\title{
PerCorsi Del Novecento
}

Maria Pacella

RESENHA Strutture dell'immaginario a cura di Rocco Mario Morano Rubbettino Editore, Soveria Mannelli (Catanzaro), 2007, 556 pp. 


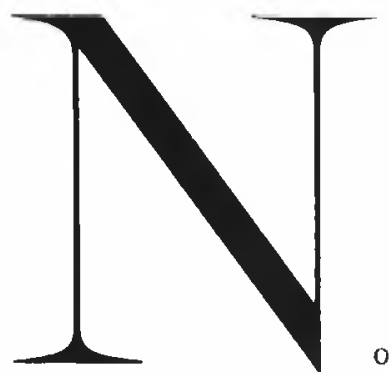

on è un caso che, alla svolta del XXI secolo gli studi e le pubblicazioni sul Novecento stiano sempre più aumentando quasi che si avesse bisogno di cambiare secolo $\mathrm{e}$ millennio per poter guardare con sguardo più esatto a quel grosso contenitore di miti, ideologie, movimenti, prodotti artistici che va sotto il nome di Novecento.

Aconferma di questo ecco uscire questo volume, Strutture dell'immaginario, Rubbettino, 2007, a cura di Rocco Mario Morano, il cui sottotitolo, Profilo del Novecento letterario italiano, rende esplicito quello che il titolo non dice dandone la giusta collocazione. Si tratta infatti degli Atti di un corso di aggiornamento per docenti di Letteratura Italiana delle Scuole Secondarie Superiori tenutosi a Cosenza fra febbraio e dicembre 2004, di fatto poi una raccolta di relazioni-saggi di 13 studiosi italiani e 3 stranieri organizzati in modo sistematico in modo tale da coprire lo sviluppo dei vari generi letterari nel secolo scorso con qualche approfondimento su questioni particolari (come la presenza di classicismi nella letteratura italiana del Novecento) e con una sezione sui reciproci rapporti influenze fra letteratura italiana e alcune letterature europee. Chiude la raccolta una stimolante e utile relazione "per una nuova didattica della letteratura del Novecento"

Tra i saggi che compongono il volume alcuni si distaccano per acutezza e profondità dell'analisi presentando anche dei veri e propri effetti di stile come il saggio di Rinaldo Rinaldi (Università di Parma) che traccia un panorama della narrativa italiana fra il 1940-45 adottando non un criterio meramente cronologico e quindi storiografico ma individuando in modo problematico alcune linee di sviluppo. Una è quella che si potrebbe chiamare dell'apertura/chiusura: alcuni autori, come d'Annunzio, Marinetti e Svevo, potrebbero essere denominati esterofili, chi per lingua, chi per nascita o formazione, scelte e gusti artistico-culturali e altri, invece, come Tozzi, sulla scia di Verga e della sua scuola, sono saldamente attaccati alla loro realtà locale; questa tensione a 
spezzare i limiti nazionali, tipica delle avanguardie, di un Savinio e di un Palazzeschi ad esempio, rinascerà poi negli anni ' 30 e ' 40 "ispirando la passione 'anglistica'e 'americanistica" di Vittorini, Fenoglio e Pavese, cioè dei più importanti narratori formatisi durante il fascismo. Un altro tema che Rinaldi individua nel romanzo della prima metà del '900 è un tema metaletterario, spesso implicito, che riguarda cioè la stessa forma-romanzo e, più precisamente, la sua dimensione. Alla tradizionale forma del romanzo lungo si oppone la forma del romanzo breve (non dimentichiamo che la brevità è una categoria moderna per eccellenza). Ed ecco le oscillazioni fra romanzo brevenovella lunga-racconto che non sono altro che un aspetto della crisi d'identità che attraversa i generi letterari nel Novecento e il romanzo in particolare. Ecco comparire i nomi di Pirandello e Landolfi anzitutto e poi Palazzeschi, Bontempelli, Calvino, Campanile, tuttili a testimoniare con le loro "invenzioni" dei vari tentativi di contrarre dilatare deformare contraffare la forma-romanzo. Come ricorda Rinaldi, "frammentare, sfarinare o granulare il romanzo significa... rinunciare a 'finirlo'... Il fantasma dell'incompiutezza e quello della parzialità di ogni frammento aleggiano sulla ricerca narrativa degli anni Venti e Trenta" (p.150). Un altro tema, questo ormai classico, della crisi del soggetto nel romanzo novecentesco viene ripreso ed esplorato con acume e finezza nelle sue diverse varianti (La coscienza di Zeno, in cui l'ironia svuota dal di dentro il protagonismo del soggetto, così come la corrispondente forma-romanzo; la Cognizione del dolore in cui l'“Io è apparentemente pieno" delle false parvenze che i ruoli e i cerimoniali sociali gli agglutinano intorno, ma in realtà lo riducono a "puro guscio vuoto"), come pure attraverso il suo rovescio nella narrativa di memoria, in cui il recupero del passato da parte di un soggetto-personaggio, spesso autobiografico, tradizionale, allontana gli spettri dell'alienazione e dell'impotenza. E qui i nomi che Rinaldi fa sono quelli di Manzini, Comisso e Bilenchi. In questo recupero del passato e dei luoghi delle origini si distingue il Vittorini di Conversazione in Sicilia (1941) in cui "l'analisi dell'Io... si realizza con l'aiuto di figure mitico-simboliche quasi degli archetipi in cui il destino individuale si riconosce" (p.157). E a questo punto viene spontaneo il rimando all'altro bel saggio di Giovanni Bárberi Squarotti (Università della Calabria) in cui, indagando la presenza della tradizione classica greco-latina nella letteratura del Novecento si dimostra, seppur riferito alla poesia, che questa tradizione è presente soprattutto come mito ridotto ad archetipo privato, fuori di ogni retorica e apparato classicistico. La letteratura del Novecento è infatti punteggiata di nomi della mitologia quasi sempre usati in modo simbolico o allegorico o parodico, in ogni caso decontestualizzati e desublimati, come relitti di un passato a cui aggrapparsi o come essenze che danno realtà all'irrealtà del presente.

Ma altri saggi e altre relazioni sarebbero degni di nota per la ricchezza delle informazioni e le stimolanti analisi. Interessanti sono per esempio i saggi di Michael Rössner (Università di Monaco) che, mettendo in relazione la letteratura italiana del Novecento e la Germania, illumina, tra l'altro, le influenze che il mondo e la cultura tedesche ebbero su Pirandello (che visse per 18 mesi a Bonn presso la cui Università si laureò e poi, in età matura, altri 3 anni a Berlino) e le sue 
opere. Anche i saggi sul teatro italiano del Novecento e quello sui rapporti fra cinema eletteratura sono notevoli per le informazioni e le utili riflessioni.

Ma alcune osservazioni si possono fare, ad esempio nel lungo saggio di Giorgio Bárberi Squarotti su "La poesia italiana dal secondo dopoguerra ai tempi più recenti" tra le decine e decine di poeti menzionati (72 per l'esattezza) solo 3 sono donne: Amelia Rosselli, a cui vengono dedicate poche righe, Maria Luisa Spaziani e Margherita Guidacci. Questo significa cancellare dalla nostra storia letteraria, specialmente da quella più recente, una presenza femminile di poeti donne importante e consolidata, anzi riconosciuta da molti come una delle caratteristiche della poesia contemporanea in Italia. Poeti rappresentativi come: Antonia Pozzi (anche se muore nel 1938, molti suoi libri sono stati pubblicati dopo il 1945), Alda Merini, Vivian Lamarque, Antonella Anedda, Iolanda Insana, Patrizia Cavalli, Mariangela Gualtieri, Patrizia Valduga, solo per fare qualche nome, presenti in varie antologie, citati in manuali e storie letterarie, sono stati del tutto ignorati. Ad Amelia Rosselli, sempre più ormai considerata poeta di primissima grandezza, come già si è detto, viene dedicato uno spazio molto esiguo. Se è vero che il tempo è esso stesso portatore di significato e permette di inquadrare persone e cose (i testi) in una luce più esatta, è altrettanto vero che ogni ricostruzione storica presenta un carattere soggettivo e individuale e che proprio per questo incorpora nella sua stessa genesi la dimensione critica, la logica della contraddizione, la possibilità, la necessità della revisione. Se a ciò si aggiunge che le scelte e le costellazioni risentono dei gusti personali del critico (maggiormente quando lo storico-critico è egli stesso autore di poesie come nel caso di Giorgio Bárberi Squarotti), ancor più evidente apparirà la legittimità di contrapporre ad un canone un altro.

Ma già questa contrapposizione è una prova dell'utilità di questo libro, della sua capacità di suscitare discussioni e porre problemi e forse è anche la dimostrazione che non è proprio vero che "il canone della letteratura italiana novecentesca si è ormai quasi del tutto cristallizzato", e che "tutto si è ordinatamente disposto in un quadro coerente, esplorato a fondo e consegnato ormai alla tradizione" come afferma Rinaldo Rinaldi (p. 141). Il quadro è meno pacificato di come appare.

Forse alcuni autori e alcuni libri aspettano ancora di essere "scoperti" per quello che sono veramente e di essere valorizzati nella loro interezza. E, tra questi, molti (o alcuni) autori donne, (oltre ad altri autori uomini, ça va sans dire).

É questa un'operazione che ci ha impegnato e che c'impegna tuttora.

Storicizzare in modo adeguato il nostro Novecento, ripercorrere il più recente passato, creare una moderna classicità, serve anche a noi, per tentare di definirci e definire l'oggi in cui viviamo. 